\title{
Improved dispersive analysis of the scalar form factor of the nucleon
}

\author{
Martin Hoferichter, ${ }^{a b}$ Christoph Ditsche, ${ }^{a}$ Bastian Kubis, ${ }^{a}$ and Ulf-G. Meißner ${ }^{a c}$ \\ ${ }^{a}$ Helmholtz-Institut für Strahlen- und Kernphysik (Theorie), Bethe Center for Theoretical \\ Physics, Universität Bonn, D-53115 Bonn, Germany \\ ${ }^{b}$ Albert Einstein Center for Fundamental Physics, Institute for Theoretical Physics, Universität \\ Bern, CH-3012 Bern, Switzerland \\ ${ }^{c}$ Forschungszentrum Jülich, Institut für Kernphysik, Jülich Center for Hadron Physics and \\ Institute for Advanced Simulation, D-52425 Jülich, Germany \\ E-mail: hoferichter@itp.unibe.ch
}

\begin{abstract}
We present a coupled system of integral equations for the $\pi \pi \rightarrow \bar{N} N$ and $\bar{K} K \rightarrow \bar{N} N S$-waves derived from Roy-Steiner equations for pion-nucleon scattering. We discuss the solution of the corresponding two-channel Muskhelishvili-Omnès problem and apply the results to a dispersive analysis of the scalar form factor of the nucleon fully including $\bar{K} K$ intermediate states. In particular, we determine the corrections $\Delta_{\sigma}$ and $\Delta_{D}$, which are needed for the extraction of the pionnucleon $\sigma$ term from $\pi N$ scattering, and show that the difference $\Delta_{D}-\Delta_{\sigma}=(-1.8 \pm 0.2) \mathrm{MeV}$ is insensitive to the input $\pi N$ parameters.
\end{abstract}

The 7th International Workshop on Chiral Dynamics, August 6 -10, 2012

Jefferson Lab, Newport News, Virginia, USA

\footnotetext{
${ }^{*}$ Speaker.
} 


\section{Introduction}

The pion-nucleon $\sigma$ term $\sigma_{\pi N}$ measures the contribution of the light quarks to the nucleon mass $m$, and is directly related to the form factor of the scalar current

$$
\sigma(t)=\frac{1}{2 m}\left\langle N\left(p^{\prime}\right)|\hat{m}(\bar{u} u+\bar{d} d)| N(p)\right\rangle, \quad t=\left(p^{\prime}-p\right)^{2}, \quad \hat{m}=\frac{m_{\mathrm{u}}+m_{\mathrm{d}}}{2},
$$

at vanishing momentum transfer $\sigma(0)=\sigma_{\pi N}$. The standard procedure for its extraction from pionnucleon $(\pi N)$ scattering relies on the low-energy theorem $[1,2]$

$$
F_{\pi}^{2} \bar{D}^{+}\left(s=m^{2}, t=2 M_{\pi}^{2}\right)=\sigma\left(2 M_{\pi}^{2}\right)+\Delta_{\mathrm{R}}
$$

which relates the Born-term-subtracted isoscalar $\pi N$ scattering amplitude at the Cheng-Dashen point $\bar{D}^{+}\left(s=m^{2}, t=2 M_{\pi}^{2}\right)$ to the scalar form factor evaluated at $2 M_{\pi}^{2}$. The remainder $\Delta_{\mathrm{R}}$ is free of chiral logarithms at full one-loop order in chiral perturbation theory (ChPT) [3, 4], and has been estimated as [3]

$$
\left|\Delta_{\mathrm{R}}\right| \lesssim 2 \mathrm{MeV}
$$

Rewriting (1.2) in terms of

$$
\Delta_{D}=F_{\pi}^{2}\left\{\bar{D}^{+}\left(s=m^{2}, t=2 M_{\pi}^{2}\right)-d_{00}^{+}-2 M_{\pi}^{2} d_{01}^{+}\right\}, \quad \Delta_{\sigma}=\sigma\left(2 M_{\pi}^{2}\right)-\sigma_{\pi N},
$$

the extraction of the $\sigma$ term reduces to the determination of the subthreshold parameters $d_{00}^{+}$and $d_{01}^{+}$as well as the combination $\Delta_{D}-\Delta_{\sigma}-\Delta_{\mathrm{R}}$. The first two corrections can be calculated using a dispersive approach [5]

$$
\Delta_{D}-\Delta_{\sigma}=(-3.3 \pm 0.2) \mathrm{MeV}
$$

where the error only covers the uncertainties in the $\pi \pi$ phase shifts available at that time. Here, we update the determination of $\Delta_{D}$ and $\Delta_{\sigma}$ using modern $\pi \pi$ phases, fully including $\bar{K} K$ intermediate states, and carefully studying the dependence of the results on $\pi N$ subthreshold parameters as well as the $\pi N$ coupling constant.

\section{Scalar pion and kaon form factors}

We first consider the case of the scalar pion and kaon form factors $F_{\pi}^{S}(t)$ and $F_{K}^{S}(t)$, which serve both to illustrate the method and as input for the scalar form factor of the nucleon. Unitarity in the $\pi \pi / \bar{K} K$ system intertwines both form factors according to [6]

$$
\operatorname{Im} F^{S}(t)=(T(t))^{*} \Sigma(t) F^{S}(t), \quad F^{S}(t)=\left(\begin{array}{c}
F_{\pi}^{S}(t) \\
\frac{2}{\sqrt{3}} F_{K}^{S}(t)
\end{array}\right)
$$

with the phase-space factor

$$
\Sigma(t)=\operatorname{diag}\left(\sigma_{t}^{\pi} \theta\left(t-t_{\pi}\right), \sigma_{t}^{K} \theta\left(t-t_{K}\right)\right), \quad \sigma_{t}^{i}=\sqrt{1-\frac{t_{i}}{t}}, \quad t_{i}=4 M_{i}^{2} \quad i \in\{\pi, K\},
$$



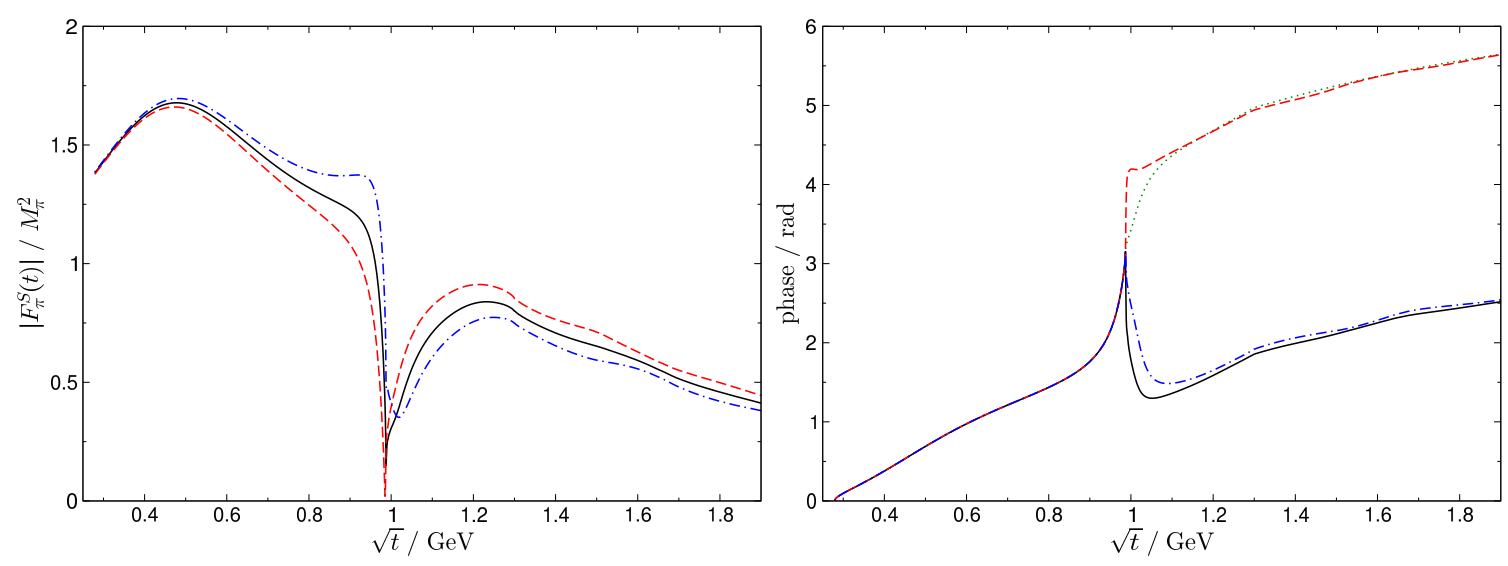

Figure 1: Modulus and phase of the scalar pion form factor. The solid, dashed, and dot-dashed lines refer to $F_{K}^{S}(0)=M_{\pi}^{2} / 2,0.4 M_{\pi}^{2}$, and $0.6 M_{\pi}^{2}$. The phase of $F_{\pi}^{S}(t)$ is compared to $\delta_{0}^{0}$, as indicated by the dotted line.

and the $T$-matrix

$$
T(t)=\left(\begin{array}{cc}
\frac{\eta_{0}^{0}(t) e^{2 i \delta_{0}^{0}(t)}-1}{2 i \sigma_{t}^{\pi}} & |g(t)| e^{i \psi_{0}^{0}(t)} \\
|g(t)| e^{i \psi_{0}^{0}(t)} & \frac{\eta_{0}^{0}(t) e^{2 i\left(\psi_{0}^{0}(t)-\delta_{0}^{0}(t)\right)}-1}{2 i \sigma_{t}^{K}}
\end{array}\right),
$$

expressed in terms of the $\pi \pi$ and $\pi \pi \rightarrow \bar{K} K$ phase shifts $\delta_{0}^{0}$ and $\psi_{0}^{0}$ as well as the inelasticity parameter $\eta_{0}^{0}=\sqrt{1-4 \sigma_{t}^{\pi} \sigma_{t}^{K}|g(t)|^{2} \theta\left(t-t_{\pi}\right)}$. The two-channel Muskhelishvili-Omnès (MO) problem $[7,8]$ defined by the unitarity relation (2.1) permits two linearly independent solutions $\Omega_{1}$, $\Omega_{2}$ [7], which may be combined in the Omnès matrix $\Omega(t)$. In general, there is no analytical solution for $\Omega(t)$, we follow here the discretization method of [9] for its numerical calculation.

Since the form factors are devoid of a left-hand cut, they are related directly to the solutions of the MO problem with coefficients determined by $F_{\pi}^{S}(0)$ and $F_{K}^{S}(0)[6]$. Using $\mathrm{ChPT}$ at $\mathscr{O}\left(p^{4}\right)$ and the low-energy constants from [10] we find

$$
F_{\pi}^{S}(0)=(0.984 \pm 0.006) M_{\pi}^{2}, \quad F_{K}^{S}(0)=(0.4 \ldots 0.6) M_{\pi}^{2},
$$

which, together with $\delta_{0}^{0}$ and $\eta_{0}^{0}$ from an extended Roy-equation analysis of $\pi \pi$ scattering [11], $\psi_{0}^{0}$ from partial-wave analyses [12], and $|g(t)|$ from a Roy-Steiner (RS) analysis of $\pi K$ scattering [13], yield the results for $F_{\pi}^{S}(t)$ depicted in Fig. 1. The strong dependence of $F_{\pi}^{S}(t)$ near $t_{K}$ on $F_{K}^{S}(0)$ attests to the inherent two-channel nature of the problem and implies that an effective single-channel description in terms of the phase of $F_{\pi}^{S}(t)$ only works for sufficiently large $F_{K}^{S}(0)$.

\section{From Roy-Steiner equations to the scalar form factor}

Unitarity couples the $\pi \pi \rightarrow \bar{N} N$ and $\bar{K} K \rightarrow \bar{N} N S$-waves $f_{+}^{0}(t)$ and $h_{+}^{0}(t)$ analogously to (2.1)

$$
\operatorname{Im} f(t)=(T(t))^{*} \Sigma(t) f(t), \quad f(t)=\left(\begin{array}{c}
f_{+}^{0}(t) \\
\frac{2}{\sqrt{3}} h_{+}^{0}(t)
\end{array}\right),
$$

but due to the presence of the left-hand cut the solution of the corresponding MO problem involves inhomogeneous contributions, which may be derived from RS equations, cf. [13-15]. Generically, 

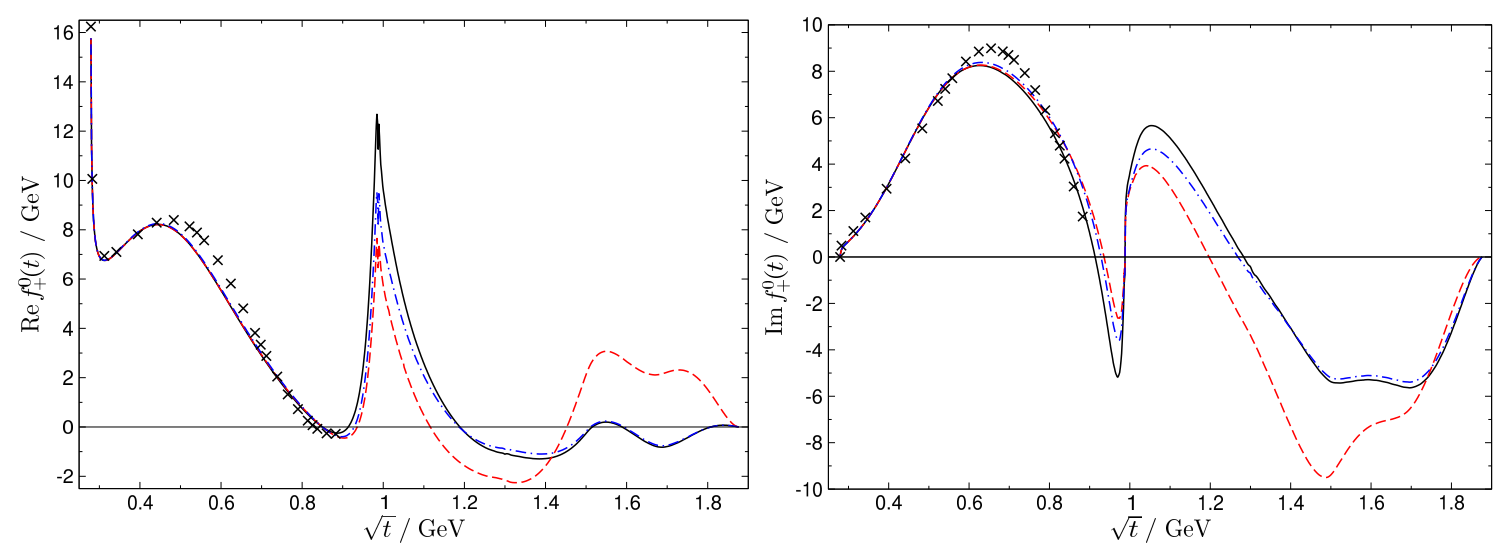

Figure 2: Results for the real and imaginary part of $f_{+}^{0}(t)$. The solid, dashed, and dot-dashed lines refer to the input RS1, RS2, and RS3 as described in the main text. The black crosses indicate the results of [17].

the integral equation takes the form

$$
f(t)=\Delta(t)+(a+b t)\left(t-4 m^{2}\right)+\frac{t^{2}\left(t-4 m^{2}\right)}{\pi} \int_{t_{\pi}}^{\infty} \mathrm{d} t^{\prime} \frac{\operatorname{Im} f\left(t^{\prime}\right)}{t^{\prime 2}\left(t^{\prime}-4 m^{2}\right)\left(t^{\prime}-t\right)},
$$

where $\Delta(t)$ includes Born terms, $s$-channel integrals, and higher $t$-channel partial waves, while $a$ and $b$ subsume subthreshold parameters that emerge as subtraction constants. The main difficulty in the evaluation of the formal solution

$$
\begin{aligned}
f(t) & =\Delta(t)+\left(t-4 m^{2}\right) \Omega(t)(\mathbb{1}-t \dot{\Omega}(0)) a+t\left(t-4 m^{2}\right) \Omega(t) b \\
& -\frac{t^{2}\left(t-4 m^{2}\right)}{\pi} \Omega(t) \int_{t_{\pi}}^{t_{\mathrm{m}}} \mathrm{d} t^{\prime} \frac{\operatorname{Im} \Omega^{-1}\left(t^{\prime}\right) \Delta\left(t^{\prime}\right)}{t^{\prime 2}\left(t^{\prime}-4 m^{2}\right)\left(t^{\prime}-t\right)}+\frac{t^{2}\left(t-4 m^{2}\right)}{\pi} \Omega(t) \int_{t_{\mathrm{m}}}^{\infty} \mathrm{d} t^{\prime} \frac{\Omega^{-1}\left(t^{\prime}\right) \operatorname{Im} f\left(t^{\prime}\right)}{t^{\prime 2}\left(t^{\prime}-4 m^{2}\right)\left(t^{\prime}-t\right)},
\end{aligned}
$$

concerns the construction of the Omnès matrix for a finite matching point $t_{\mathrm{m}}$ [14].

In the numerical analysis we put $\operatorname{Im} f(t)=0$ above $t_{\mathrm{m}}$, which we choose as $t_{\mathrm{m}}=4 \mathrm{~m}^{2}$ (thus exploiting a kinematical zero of $f(t)$ ), take the $\pi N$ and $K N s$-channel partial waves from [16], and use the KH80 $\pi N$ coupling constant and subthreshold parameters as reference point [17]. In order to assess the uncertainties for higher energies we consider the following variants of the input: first, we keep the phase shifts $\delta_{0}^{0}$ and $\psi_{0}^{0}$ constant above $\sqrt{t_{0}}=1.3 \mathrm{GeV}$ ("RS1"), where $4 \pi$ intermediate states become important and thus the two-channel approximation will break down, and second, guide both phase shifts smoothly to their asymptotic value of $2 \pi$ as for the meson form factors ("RS2"). Finally, we amend RS1 in such a way that $\Delta_{2}(t)$, the $K N$ component of the inhomogeneity, is put to zero in order to assess the uncertainty in the $K N$ input ("RS3"). The corresponding results for $f_{+}^{0}(t)$ depicted in Fig. 2 show that the largest uncertainty is induced by the high-energy phase shifts.

\section{Results}

The scalar form factor of the nucleon fulfills the unitarity relation

$$
\operatorname{Im} \sigma(t)=\frac{2}{4 m^{2}-t}\left\{\frac{3}{4} \sigma_{t}^{\pi}\left(F_{\pi}^{S}(t)\right)^{*} f_{+}^{0}(t) \theta\left(t-t_{\pi}\right)+\sigma_{t}^{K}\left(F_{K}^{S}(t)\right)^{*} h_{+}^{0}(t) \theta\left(t-t_{K}\right)\right\}
$$



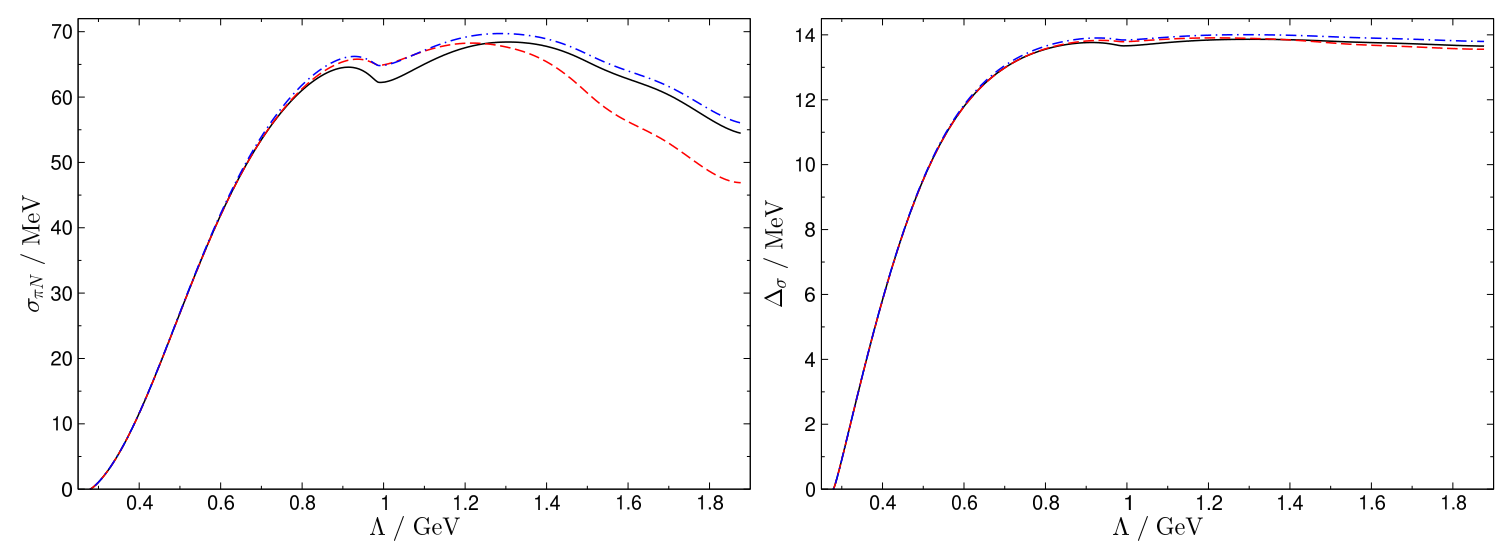

Figure 3: $\sigma_{\pi N}$ and $\Delta_{\sigma}$ as a function of the integral cutoff $\Lambda$.

so that, based on the results of the previous sections, the un- and once-subtracted dispersion relations

$$
\sigma(t)=\frac{1}{\pi} \int_{t_{\pi}}^{\infty} \mathrm{d} t^{\prime} \frac{\operatorname{Im} \sigma\left(t^{\prime}\right)}{t^{\prime}-t}=\sigma_{\pi N}+\frac{t}{\pi} \int_{t_{\pi}}^{\infty} \mathrm{d} t^{\prime} \frac{\operatorname{Im} \sigma\left(t^{\prime}\right)}{t^{\prime}\left(t^{\prime}-t\right)}
$$

evaluated at $t=0$ and $t=2 M_{\pi}^{2}$ in principle determine $\sigma_{\pi N}$ and $\Delta_{\sigma}$ provided the two-channel approximation for the spectral function is sufficiently accurate in the energy range dominating the dispersive integral. Fig. 3 shows that, while the dispersion relation converges too slowly for the $\sigma$ term itself, the result for $\Delta_{\sigma}$ becomes stable for $\Lambda \gtrsim 1 \mathrm{GeV}$. Adding the uncertainties from the spectral function and the variation of the integral cutoff between $\Lambda=1.3 \mathrm{GeV}$ and $\Lambda=2 \mathrm{~m}$, we find

$$
\begin{aligned}
\Delta_{\sigma} & =(13.9 \pm 0.3) \mathrm{MeV} \\
& +Z_{1}\left(\frac{g^{2}}{4 \pi}-14.28\right)+Z_{2}\left(d_{00}^{+} M_{\pi}+1.46\right)+Z_{3}\left(d_{01}^{+} M_{\pi}^{3}-1.14\right)+Z_{4}\left(b_{00}^{+} M_{\pi}^{3}+3.54\right), \\
Z_{1} & =0.36 \mathrm{MeV}, \quad Z_{2}=0.57 \mathrm{MeV}, \quad Z_{3}=12.0 \mathrm{MeV}, \quad Z_{4}=-0.81 \mathrm{MeV},
\end{aligned}
$$

where we have made the dependence on the $\pi N$ parameters explicit (note that more modern determinations point to lower values of the $\pi N$ coupling constant around $g^{2} / 4 \pi \sim 13.7$ [18-20]).

Similarly, the correction $\Delta_{D}$ follows from the $t$-channel expansion

$$
\bar{D}^{+}\left(s=m^{2}, t\right)=d_{00}^{+}+d_{01}^{+} t-16 t^{2} \int_{t_{\pi}}^{\infty} \mathrm{d} t^{\prime} \frac{\operatorname{Im} f_{+}^{0}\left(t^{\prime}\right)}{t^{\prime 2}\left(t^{\prime}-4 m^{2}\right)\left(t^{\prime}-t\right)}+\{J \geq 2\}+\{s \text {-channel integrals }\}
$$

evaluated at $t=2 M_{\pi}^{2}$, which gives

$$
\begin{aligned}
\Delta_{D} & =(12.1 \pm 0.3) \mathrm{MeV} \\
& +\tilde{Z}_{1}\left(\frac{g^{2}}{4 \pi}-14.28\right)+\tilde{Z}_{2}\left(d_{00}^{+} M_{\pi}+1.46\right)+\tilde{Z}_{3}\left(d_{01}^{+} M_{\pi}^{3}-1.14\right)+\tilde{Z}_{4}\left(b_{00}^{+} M_{\pi}^{3}+3.54\right), \\
\tilde{Z}_{1} & =0.42 \mathrm{MeV}, \quad \tilde{Z}_{2}=0.67 \mathrm{MeV}, \quad \tilde{Z}_{3}=12.0 \mathrm{MeV}, \quad \tilde{Z}_{4}=-0.77 \mathrm{MeV} .
\end{aligned}
$$


Comparison with (4.3) shows that the dependence on the $\pi N$ parameters cancels nearly completely in the difference

$$
\Delta_{D}-\Delta_{\sigma}=(-1.8 \pm 0.2) \mathrm{MeV} .
$$

This cancellation can be explained by the observation that the spectral function in both dispersion relations involves $f_{+}^{0}$ in a very similar manner, so that both integrals are equally affected by the dependence on the $\pi N$ parameters inherited from $f_{+}^{0}$. In the same way, part of the uncertainties discussed in Sect. 3 drop out, so that the final error estimate for $\Delta_{D}-\Delta_{\sigma}$ even decreases compared to the uncertainty in both corrections individually.

\section{References}

[1] T. P. Cheng and R. F. Dashen, Phys. Rev. Lett. 26 (1971) 594.

[2] L. S. Brown, W. J. Pardee and R. D. Peccei, Phys. Rev. D 4 (1971) 2801.

[3] V. Bernard, N. Kaiser and U.-G. Meißner, Phys. Lett. B 389 (1996) 144 [hep-ph/9607245].

[4] T. Becher and H. Leutwyler, JHEP 0106 (2001) 017 [hep-ph/0103263].

[5] J. Gasser, H. Leutwyler and M. E. Sainio, Phys. Lett. B 253 (1991) 260.

[6] J. F. Donoghue, J. Gasser and H. Leutwyler, Nucl. Phys. B 343 (1990) 341.

[7] N. I. Muskhelishvili, Singular Integral Equations, Wolters-Noordhoff Publishing, Groningen, 1953.

[8] R. Omnès, Nuovo Cim. 8 (1958) 316.

[9] B. Moussallam, Eur. Phys. J. C 14 (2000) 111 [hep-ph/9909292].

[10] G. Colangelo et al., Eur. Phys. J. C 71 (2011) 1695 [arXiv:1011.4408 [hep-lat]].

[11] I. Caprini, G. Colangelo and H. Leutwyler, Eur. Phys. J. C 72 (2012) 1860 [arXiv:1111.7160 [hep-ph]]; in preparation.

[12] D. H. Cohen, D. S. Ayres, R. Diebold, S. L. Kramer, A. J. Pawlicki and A. B. Wicklund, Phys. Rev. D 22 (1980) 2595; A. Etkin et al., Phys. Rev. D 25 (1982) 1786.

[13] P. Büttiker, S. Descotes-Genon and B. Moussallam, Eur. Phys. J. C 33 (2004) 409 [hep-ph/0310283].

[14] C. Ditsche, M. Hoferichter, B. Kubis and U.-G. Meißner, JHEP 1206 (2012) 043 [arXiv:1203.4758 [hep-ph]]; JHEP 1206 (2012) 063 [arXiv:1204.6251 [hep-ph]].

[15] M. Hoferichter, D. R. Phillips and C. Schat, Eur. Phys. J. C 71 (2011) 1743 [arXiv:1106.4147 [hep-ph]].

[16] R. A. Arndt, W. J. Briscoe, I. I. Strakovsky, and R. L. Workman, Eur. Phys. J. A 35 (2008) 311; J. S. Hyslop, R. A. Arndt, L. D. Roper, and R. L. Workman, Phys. Rev. D 46 (1992) 961.

[17] G. Höhler, Pion-Nukleon-Streuung: Methoden und Ergebnisse, in Landolt-Börnstein, 9b2, ed. H. Schopper, Springer Verlag, Berlin, 1983.

[18] J. J. de Swart, M. C. M. Rentmeester and R. G. E. Timmermans, PiN Newslett. 13 (1997) 96 [nucl-th/9802084].

[19] R. A. Arndt, W. J. Briscoe, I. I. Strakovsky and R. L. Workman, Phys. Rev. C 74 (2006) 045205 [nucl-th/0605082].

[20] V. Baru, C. Hanhart, M. Hoferichter, B. Kubis, A. Nogga and D. R. Phillips, Phys. Lett. B 694 (2011) 473 [arXiv:1003.4444 [nucl-th]]; Nucl. Phys. A 872 (2011) 69 [arXiv:1 107.5509 [nucl-th]]. 